\title{
Traffic Grooming in Unidirectional WDM Rings with Bounded Degree Request Graph`
}

\author{
Xavier Muñoz ${ }^{1}$ and Ignasi Sau ${ }^{1,2}$ \\ ${ }^{1}$ Graph Theory and Combinatorics Group at Applied Mathematics IV \\ Department of UPC - Barcelona, Spain \\ xml@ma4.upc. edu \\ ${ }^{2}$ Mascotte joint Project - INRIA/CNRS-I3S/UNSA - 2004 \\ route des Lucioles - Sophia-Antipolis, France \\ Ignasi.Sau@sophia.inria.fr
}

\begin{abstract}
Traffic grooming is a major issue in optical networks. It refers to grouping low rate signals into higher speed streams, in order to reduce the equipment cost. In SONET WDM networks, this cost is mostly given by the number of electronic terminations, namely Add-Drop Multiplexers (ADMs for short). We consider the unidirectional ring topology with a generic grooming factor $C$, and in this case, in graph-theoretical terms, the traffic grooming problem consists in partitioning the edges of a request graph into subgraphs with at most $C$ edges, while minimizing the total number of vertices of the decomposition.

We consider the case when the request graph has bounded degree $\Delta$, and our aim is to design a network (namely, place the ADMs at each node) being able to support any request graph with maximum degree at most $\Delta$. The existing theoretical models in the literature are much more rigid, and do not allow such adaptability. We formalize the problem, and we solve the cases $\Delta=2$ (for all values of $C$ ) and $\Delta=3$ (except the case $C=4)$. We also provide lower and upper bounds for the general case.
\end{abstract}

Keywords: optical networks, SONET over WDM, traffic grooming, ADM, graph decomposition, cubic graph, bridgeless graph.

\section{Introduction}

Traffic grooming is the generic term for packing low rate signals into higher speed streams (see the surveys $3,9,16,18,22$ ). By using traffic grooming, it is possible to bypass the electronics at the nodes which are not sources or destinations of traffic, and therefore reducing the cost of the network. Typically, in a WDM (Wavelength Division Multiplexing) network, instead of having one SONET Add

* This work has been partially supported by European project IST FET AEOLUS, PACA region of France, Ministerio de Educación y Ciencia of Spain, European Regional Development Fund under project TEC2005-03575, Catalan Research Council under project 2005SGR00256, and COST action 293 GRAAL, and has been done in the context of the CRC CORSO with France Telecom.

H. Broersma et al. (Eds.): WG 2008, LNCS 5344, pp. 300 3112008.

(C) Springer-Verlag Berlin Heidelberg 2008 
Drop Multiplexer (ADM) on every wavelength at every node, it may be possible to have ADMs only for the wavelengths used at that node (the other wavelengths being optically routed without electronic switching).

The so called traffic grooming problem consists in minimizing the total number of ADMs to be used, in order to reduce the overall cost of the network. The problem is easily seen to be NP-complete for an arbitrary set of requests. See [1,11,12] for hardness and approximation results of traffic grooming in rings, trees and star networks.

Here we consider unidirectional SONET/WDM ring networks. In that case the routing is unique and we have to assign to each request between two nodes a wavelength and some bandwidth on this wavelength. If the traffic is uniform and if a given wavelength can carry at most $C$ requests, we can assign to each request at most $\frac{1}{C}$ of the bandwidth. $C$ is known as the grooming ratio or grooming factor. Furthermore if the traffic requirement is symmetric, it can be easily shown (by exchanging wavelengths) that there always exists an optimal solution in which the same wavelength is given to a pair of symmetric requests. Then without loss of generality we will assign to each pair of symmetric requests, called a circle, the same wavelength. Then each circle uses $\frac{1}{C}$ of the bandwidth in the whole ring. If the two end-nodes are $i$ and $j$, we need one ADM at node $i$ and one at node $j$. The main point is that if two requests have a common end-node, they can share an ADM if they are assigned the same wavelength.

The traffic grooming problem for a unidirectional SONET ring with $n$ nodes and a grooming ratio $C$ has been modeled as a graph partition problem in both [2] and [15] when the request graph is given by a symmetric graph $R$. To a wavelength $\lambda$ is associated a subgraph $B_{\lambda} \subset R$ in which each edge corresponds to a pair of symmetric requests (that is, a circle) and each node to an ADM. The grooming constraint, i.e. the fact that a wavelength can carry at most $C$ requests, corresponds to the fact that the number of edges $\left|E\left(B_{\lambda}\right)\right|$ of each subgraph $B_{\lambda}$ is at most $C$. The cost corresponds to the total number of vertices used in the subgraphs involved in the partition, and the objective is therefore to minimize this number.

This problem has been well studied when the network is a unidirectional ring $3,4,7,8,9,13,15,14,16,20,21$. With the all-to-all set of requests, optimal constructions for a given grooming ratio $C$ were obtained using tools of graph and design theory, in particular for grooming ratio $C=3,4,5,6$ and $C \geq N(N-$ 1) $/ 6$ [3.

Most of the research efforts in this grooming problem have been devoted to finding the minimum number of ADMs required either for a given set of connection requests (typically a uniform all-to-all communication pattern) or for a general traffic pattern. In all this articles the traffic pattern has been considered as an input for the problem of placing the ADMs. In this paper we consider the traffic grooming problem from a different point of view: assuming a given network topology, it would be desirable to place the minimum number of ADMs as possible at each node in such a way that they could be configured 
to handle different traffic patterns or graphs of requests. One cannot expect to change the equipment of the network each time the traffic requirements change.

Without any restriction on the graph of requests, the number of required $\mathrm{ADMs}$ is given by the worst case, i.e. when the Graph of Requests is the complete graph. However, in many cases some restrictions on the graph of requests might be assumed. From a practical point of view, it is interesting to design a network being able to support any request graph with maximum degree not exceeding a given constant. This situation is usual in real optical networks, since due to technology constraints the number of allowed communications for each node is usually bounded. This flexibility can also be thought from another point of view: if we have a limited number of available ADMs to place at the nodes of the network, then it is interesting to know which is the maximum degree of a request graph that our network is able to support, depending on the grooming factor. Equivalently, given a maximum degree and a number of available ADMs, it is useful to know which values of the grooming factor the network will support.

The aim of this article is to provide a theoretical framework to design such networks with dynamically changing traffic. We study the case when the physical network is given by an unidirectional ring, which is a widely used topology (for instance, SONET rings). In [6] the authors consider this problem from a more practical point of view: they call $t$-allowable a traffic matrix where the number of circuits terminated at each node is at most $t$, and the objective is also to minimize the number of electronic terminations. They give lower bounds on the number of ADMs and provide some heuristics.

In addition, we also suppose that each pair of communicating nodes establishes a two-way communication. That is, each pair $(i, j)$ of communicating nodes in the ring represents two requests: from $i$ to $j$, and from $j$ to $i$. Thus, such a pair uses all the edges of the ring, therefore inducing one unity of load. Hence, we can use the notation introduced in 4 and consider each request as an edge, and then again the grooming constraint, i.e. the fact that a wavelength can carry at most $C$ requests, corresponds to the fact that the number of edges $\left|E\left(B_{\lambda}\right)\right|$ of each subgraph $B_{\lambda}$ is at most $C$. The cost corresponds to the total number of vertices used in the subgraphs involved in the partition.

Namely, we consider the problem of placing the minimum number of ADMs in the nodes of the ring in such a way that the network could support any request graph with maximum degree bounded by a constant $\Delta$. Note that using this approach, as far as the degree of each node does not exceed $\Delta$, the network can support a wide range of traffic demands without the installation of additional electronic switches at the nodes. The problem can be formally stated as follows: Traffic Grooming in Unidirectional Rings with Bounded-Degree REQUest Graph

Input: Three integers $n, C$, and $\Delta$.

Output: An assignment of $A(v) \mathrm{ADMs}$ to each node $v \in V\left(C_{n}\right)$, in such a way that for any undirected request graph $R$ with maximum degree at most $\Delta$, it exists a partition of $E(R)$ into subgraphs $B_{\lambda}, 1 \leq \lambda \leq \Lambda$, such that: 
(i) $\left|E\left(B_{\lambda}\right)\right| \leq C$ for all $\lambda$; and

(ii) each vertex $v \in V\left(C_{n}\right)$ appears in at most $A(v)$ subgraphs.

Objective: Minimize $\sum_{v \in V\left(C_{n}\right)} A(v)$, and the optimum is denoted $A(n, C, \Delta)$.

When the request graph is restricted to belong to a subclass of graphs $\mathcal{C}$ of the class of graphs with maximum degree at most $\Delta$, then the optimum is denoted $A(n, C, \Delta, \mathcal{C})$. Obviously, for any subclass of graph $\mathcal{C}, A(n, C, \Delta, \mathcal{C}) \leq A(n, C, \Delta)$.

In this article we solve the cases corresponding to $\Delta=2$ and $\Delta=3$ (giving a conjecture for the case $C=4$ ), and give lower bounds for the general case. The remainder of the article is structured as follows: in Section 2 we give some properties of the function $A(n, C, \Delta)$, to be used in the following sections. In Section 3 we focus on the case $\Delta=2$, giving a closed formula for all values of $C$. In Section 4 we study the case $\Delta=3$, solving all cases except the case $C=4$, for which we conjecture the solution. Finally, Section 5 is devoted to conclusions and open problems.

\section{Properties of $A(n, C, \Delta)$}

In this section we describe some properties of the function $A(n, C, \Delta)$.

Lemma 1. The following statements hold:

(i) $A(n, C, 1)=n$.

(ii) $A(n, 1, \Delta)=\Delta n$.

(iii) If $C^{\prime} \geq C$, then $A\left(n, C^{\prime}, \Delta\right) \leq A(n, C, \Delta)$.

(iv) If $\Delta^{\prime} \geq \Delta$, then $A\left(n, C, \Delta^{\prime}\right) \geq A(n, C, \Delta)$.

(v) $A(n, C, \Delta) \geq n$ for all $\Delta \geq 1$.

(vi) If $C \geq \frac{n \Delta}{2}, A(n, C, \Delta)=n$.

Proof. (i) The request graph can consist in a perfect matching, so any solution uses 1 ADM per node.

(ii) A $\Delta$-regular graph can be partitioned into $\frac{n \Delta}{2}$ disjoint edges.

(iii) Any solution for $C$ is also a solution for $C^{\prime}$.

(iv) If $\Delta^{\prime} \geq \Delta$, the subgraphs with maximum degree at most $\Delta$ are a subclass of the class of graphs with maximum degree at most $\Delta^{\prime}$.

(v) Combine (i) and (iv).

(vi) In this case all the edges of the request graph fit into one subgraph.

Since we are interested in the number of ADMs required at each node, let us consider the following definition:

Definition 1. Let $M(C, \Delta)$ be the least positive number $M$ such that, for any $n \geq 1$, the inequality $A(n, C, \Delta) \leq M n$ holds.

Lemma 2. $M(C, \Delta)$ is a natural number.

Proof. First of all, we know by Lemma 1 that, for any $C \geq 1, A(n, C, \Delta) \leq$ $A(n, 1, \Delta)=\Delta n$. Thus $A(n, C, \Delta)$ is upper-bounded by $\Delta n$. On the other hand, since any vertex may appear in the request graph, $A(n, C, \Delta)$ is lower-bounded 
by $n$. Suppose now that $M$ is not a natural number. That is, suppose that $r<M<r+1$ for some positive integer $r$. Therefore, there must be at least $(r+1-M) n$ vertices with at most $r$ ADMs each. For each $n$, let $V_{n, r}$ be the subset of vertices of the request graph with at most $r$ ADMs. Then, since $r+1-M>0$, we have that $\lim _{n \rightarrow \infty}\left|V_{n, r}\right|=\infty$. In other words, there is an arbitrarily big subset of vertices with at most $r$ ADMs per vertex. But we can consider a request graph with maximum degree at most $\Delta$ on the set of vertices $V_{n, r}$, and this means that $r$ ADMs per node is enough, a contradiction with the optimality of $M$.

If the request graph is restricted to belong to a subclass of graphs $\mathcal{C}$ of the class of graphs with maximum degree at most $\Delta$, then the corresponding positive integer is denoted $M(C, \Delta, \mathcal{C})$. Again, for any subclass $\mathcal{C}, M(C, \Delta, \mathcal{C}) \leq M(C, \Delta)$.

We provide now a lower bound on $M(C, \Delta)$.

Proposition 1 (General Lower Bound). $M(C, \Delta) \geq\left\lceil\frac{C+1}{C} \frac{\Delta}{2}\right\rceil$.

Proof. Erdös and Sachs [10] proved that for any integer $k$ there exist $k$-regular graphs with arbitrary large girth. For each value of $C$ and $\Delta$, let $G$ be a $\Delta$ regular graph with girth at least $C+1$, and let $n=|V(G)|$. Clearly all the subgraphs (with at most $C$ edges) involved of the partition of the $\Delta n / 2$ edges of $G$ are trees. Therefore, the total number of vertices of any partition is at least $\frac{\Delta(C+1)}{2 C} n$ (this can be easily seen using that the function $(x+1) / x$ with $1 \leq x \leq C$ is minimized when $x=C$ ). Then necessarily a vertex must occur in at least $\frac{\Delta(C+1)}{2 C}$ subgraphs, yielding the desired bound.

Let a $\Delta$-graph be a graph with maximum degree at most $\Delta$. By Lemma 2 , $A(n, C, \Delta)$ is of the form $A(n, C, \Delta)=M(C, \Delta) n-\alpha(C, \Delta)$, where $M(C, \Delta)$ and $\alpha(C, \Delta)$ are natural numbers depending only on $C$ and $\Delta$. Since the network must be designed in order to support any $\Delta$-graph, if there exists a $\Delta$-graph $H$ that requires strictly more than $M(C, \Delta) \mathrm{ADMs}$ at some vertex of the network, then by considering the same graph $H$ on different subsets of vertices of the network, we could force at least $M(C, \Delta)+1 \mathrm{ADMs}$ in $\Omega(n)$ nodes of the network, which would be in contradiction with the definition of $M(C, \Delta)$. That is, each vertex can appear in at most $M(C, \Delta)$ subgraphs.

In other words, for each value of $C$ and $\Delta$, the problem reduces to finding the least positive integer $M$ such that the edges of any $\Delta$-graph can be partitioned into subgraphs with at most $C$ edges, in such a way that each vertex appears in at most $M$ subgraphs.

Due to the above discussion, in the sequel we focus on determining the parameter $M(C, \Delta)$ for each value of $C$ and $\Delta$. Observe also that any $\Delta$-graph $H$ is a subgraph of some $\Delta$-regular graph $G$ (with possible more vertices). Note also that if we restrict a partition of $G$ to the vertices of $H$, the number of occurrences of the vertices cannot increase. Said otherwise, to determine the value of $M(C, \Delta)$ it is enough to consider $\Delta$-regular graphs.

Lemma 3. The following statements hold trivially: 
(i) $M(C, 1)=1$ for all $C \geq 1$.

(ii) $M(1, \Delta)=\Delta$ for all $\Delta \geq 1$.

(iii) If $C^{\prime} \geq C$, then $M\left(C^{\prime}, \bar{\Delta}\right) \leq M(C, \Delta)$.

(iv) If $\Delta^{\prime} \geq \Delta$, then $M\left(C, \Delta^{\prime}\right) \geq M(C, \Delta)$.

(v) $M(C, \Delta) \leq \Delta$ for all $C, \Delta \geq 1$.

\section{Case $\Delta=2$}

In this case we provide not only the value of $M(C, 2)$, but also the exact expression of the cost function $A(n, C, 2)$.

Proposition 2. $A(n, C, 2)=2 n-(C-1)$.

Proof. Consider the case when the request graph is 2-regular and has girth greater than $C$ (such a graph exists by [10]). Then, a feasible solution is obtained by placing $2 \mathrm{ADMs}$ at each vertex. We count in how many vertices we can assure that we can place only one ADM.

Let us see first that we cannot use $1 \mathrm{ADM}$ in more than $C-1$ vertices. Suppose this, i.e. that we have $1 \mathrm{ADM}$ in $C$ vertices and 2 in all the others. Then, consider a set of requests given by a cycle $H$ of length $C+1$ containing all the $C$ vertices with $1 \mathrm{ADM}$ inside it, and other cycles containing the remaining vertices. In this situation, we are forced to use 2 subgraphs for the vertices of $H$, and at least 2 vertices of $H$ must appear in both subgraphs. Hence we will need more than 1 $\mathrm{ADM}$ in some vertex that had initially only $1 \mathrm{ADM}$.

Now, let us see that we can always save $C-1$ ADMs. Let $\left\{a_{0}, a_{1}, \ldots, a_{C-2}\right\}$ be the set of vertices with only $1 \mathrm{ADM}$, that we can choose arbitrarily. We will see that we can decompose the set of requests in such a way that the vertices $a_{i}$ always appear with degree 2 in some subgraph, covering in this way both requests of each vertex with only $1 \mathrm{ADM}$. Indeed, suppose first that two of these vertices (namely, $a_{i}$ and $a_{j}$ ) do not appear consecutively in one of the disjoint cycles of the set of requests. Let $b_{i}$ be the nearest vertex to $a_{i}$ in the cycle in the direction of $a_{j}$, and conversely for $b_{j}$ ( $b_{i}$ may be equal to $b_{j}$ if $a_{i}$ and $a_{j}$ are separated by exactly one vertex). Then, consider two paths (eventually, cycles) of the form $\left\{b_{i}, a_{i}, \ldots\right\}$ and $\left\{b_{j}, a_{j}, \ldots\right\}$, to assure that both $a_{i}$ and $a_{j}$ lie in the middle of the subgraph. We do the same construction for each pair of non-consecutive vertices.

Now, consider all the vertices $\left\{a_{0}, \ldots, a_{i}, \ldots, a_{t-1}\right\}$ which are adjacent in the same cycle of the request graph, with $t \leq C-1$. Let $b_{0}$ be the nearest vertex to $a_{0}$ different from $a_{1}$, and let $b_{t-1}$ be the nearest vertex to $a_{t-1}$ different from $a_{t-2}$. Then, consider a subgraph with the path (or cycle, if $b_{0}=b_{t-1}$ ) $\left\{b_{0} a_{0} a_{1} \ldots a_{t-1} b_{t-1}\right\}$.

\section{Case $\Delta=3$}

We study the cases $C=3$ and $C \geq 5$ in Sections 4.1 and 4.2 , respectively. We discuss the open case $C=4$ in Section $[5$ 


\subsection{Case $C=3$}

We study first the case when the request graph is a bridgeless cubic graph, and then the case of a general request graph.

Bridgeless Cubic Request Graph. We will need some preliminary graph theoretical concepts. Let $G=(V, E)$ be a graph. For $A, B \subseteq V$, an $A-B$ path in $G$ is a path from $x$ to $y$, with $x \in A$ and $y \in B$.

If $A, B \subseteq V$ and $X \subseteq V \cup E$ are such that every $A$ - $B$ path in $G$ contains a vertex or an edge from $X$, we say that $X$ separates the sets $A$ and $B$ in $G$. More generally we say that $X$ separates $G$ if $G-X$ is disconnected, that is, if $X$ separates in $G$ some two vertices that are not in $X$. A separating set of vertices is a separator.

A vertex which separates two other vertices of the same component is a cutvertex, and an edge separating its ends is a bridge. Thus, the bridges in a graph are precisely those edges that do not lie on any cycle. A set $M$ of independent edges in a graph $G=(V, E)$ is called a matching. A matching that covers all the vertices in $V$ is called perfect. A $k$-regular spanning subgraph is called a $k$-factor. Thus, a subgraph $H \subseteq G$ is a 1 -factor of $G$ if and only if $E(H)$ is a perfect matching of $V$. Such a matching is also called perfect matching.

We recall a well known result from matching theory proved by Petersen in 1891 [17:

Theorem 1 ( [17]). Every bridgeless cubic graph has a 1-factor.

If we remove a 1-factor from a cubic graph, what it remains is a disjoint set of cycles.

Corollary 1. Every bridgeless cubic graph has a decomposition into a 1-factor and disjoint cycles.

An example of a decomposition of a bridgeless cubic graph into disjoints cycles and a 1-factor is depicted in FIG. 1h.

Proposition 3. Let $\mathcal{C}$ be the class of cubic graphs with a perfect matching. Then,

$$
M(3,3, \mathcal{C})=2 .
$$

Proof. Let us proof that we can always partition the request graph into paths with 3 edges in such a way that each vertex appears in 2 paths. To do so, we take the decomposition given by Proposition 1, together with a clockwise orientation of the edges of each cycle. With this orientation, each edge of the 1-factor has two incoming and two outgoing edges of the cycles. For each edge of the 1-factor we take its two incoming edges, and form in this way a path of length 3 . It is easy to verify that this is indeed a decomposition into paths of length three. For instance, if we do this construction in the graph of FIG. [at, and we label the edges of the 1 -factor as $\{A, B, \ldots, G\}$ and the ones of the cycles as $\{1,2, \ldots, 14\}$ (see FIG. 1b), we obtain the following decomposition:

$$
\{1, A, 6\},\{5, B, 2\},\{3, C, 8\},\{7, D, 9\},\{14, E, 11\},\{10, F, 12\},\{4, G, 13\}
$$




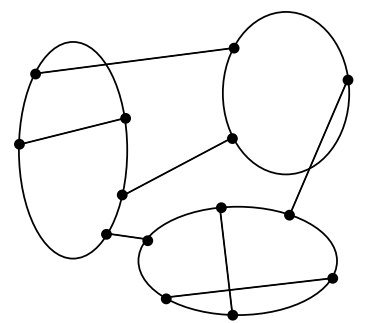

a)

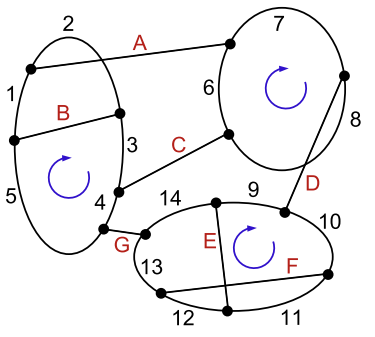

b)

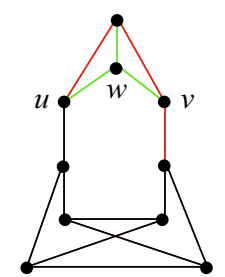

c)

Fig. 1. a) Decomposition of a bridgeless cubic graph into disjoints cycles and a 1factor. b) Decomposition of a bridgeless cubic graph into paths of length 3. c) Cubic bridgeless graph used in the proof of Proposition 3

Now let us see that we cannot do better, i.e. with $2 n-1$ ADMs. If such a solution exists, there would be at least one vertex with only $1 \mathrm{ADM}$, and the average of the number of ADMs of all the other vertices must not exceed 2. In order to see that this is not always possible, consider the cubic bridgeless graph on 10 vertices of Fig. 1k. Let $w$ be the vertex with only 1 ADM. This graph has no triangles except those containing $w$. Since we can use only 1 ADM in $w$, we must take all its requests in one subgraph. It is not possible to cover the 4 remaining requests of the nodes $u$ and $v$ in one subgraph, and thus without loss of generality we will need 3 ADMs in $u$. With these constraints, one can check that the best solution uses 20 ADMs, that is $2 n>2 n-1$.

General Request Graph. It turns out that when the request graph is not restricted to be bridgeless we have that $M(3,3)=3$.

Proposition 4. $M(3,3)=3$.

Proof. By (ii) and (iii) of Lemma 3 we know that $M(3,3) \leq 3$. We shall exhibit a counterexample showing that $M(3,3)>2$, proving the result. Consider the cubic graph $G$ depicted in FIG. 2 . We will prove that it is not possible to partition the edges of $G$ into subgraphs with at most 3 edges in such a way that each vertex appears in at most 2 subgraphs.

Indeed, suppose the opposite, i.e. that we can partition the edges of $G$ into subgraphs $B_{1}, \ldots, B_{k}$ with $\left|E\left(B_{i}\right)\right| \leq 3$ in such a way that each vertex appears in at most 2 subgraphs, and let us reach a contradiction.

Following the notation illustrated in FIG. $2 \mathrm{a}$, let $A_{1}, A_{2}, A_{3}$ be the connected components of $G \backslash\left\{e_{1}, e_{2}, e_{3}\right\}$. Let also, with abuse of notation, $a_{i}=A_{i} \cap e_{i}$, $i=1,2,3$, and $a_{0}=e_{1} \cap e_{2} \cap e_{3}$.

Claim. There exist an index $i^{*} \in\{1,2,3\}$ and a subgraph $B_{k^{*}}$ containing $a_{0}$, such that $B_{k^{*}} \cap A_{i^{*}}=\left\{a_{i *}\right\}$.

Proof. Among all the subgraphs $B_{1}, \ldots, B_{k}$ involved in the decomposition of $G$, consider the $\ell$ subgraphs $B_{j_{1}}, \ldots, B_{j_{\ell}}$ covering the edges $\left\{e_{1}, e_{2}, e_{3}\right\}$. If $\ell=$ 1 , then the subgraph $B_{j_{1}}$ is a star with three edges and center $a_{0}$, and then 


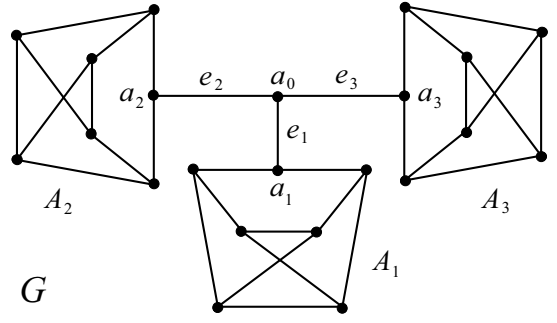

a)

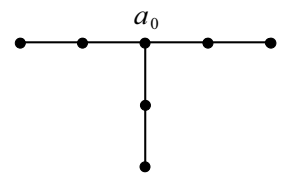

b)

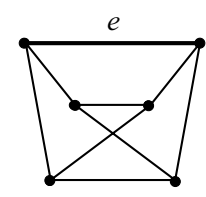

$H$

c)

Fig. 2. a) Cubic graph $G$ that can not be edge-partitioned into subgraphs with at most 3 edges in such a way that each vertex appears in at most 2 subgraphs. b) Graph that cannot be partitioned into 2 connected subgraphs with at most 3 edges. c) Counterexample of Proposition 4 showing that $M(3,3)=3$.

$B_{j_{1}} \cap A_{i}=\left\{a_{i}\right\}$ for each $i=1,2,3$. If $\ell \geq 3$, then the vertex $a_{0}$ appears in 3 subgraphs, a contradiction. Hence it remains to handle the case $\ell=2$. If the claim was not true, it would imply that for each $i=1,2,3$ it would exist $j_{f(i)} \in\left\{j_{1}, j_{2}\right\}$ such that $B_{f(i)} \cap A_{i}$ contains at least one edge. In particular, this would imply that the graph depicted in FIG. 2 $\mathrm{b}$ could be partitioned into two connected subgraphs with at most three edges, which is clearly not possible.

Suppose without loss of generality that the index $i^{*}$ given by Claim 4.1 is equal to 1 . Thus, $a_{1}$ appears in a subgraph $B_{k^{*}}$ that does not contain any edge of $A_{1}$. Therefore, the edges of $A_{1}$ must be partitioned into connected subgraphs with at most 3 edges, in such a way that $a_{1}$ appears in only 1 subgraph, and all its other vertices in at most 2 subgraphs, each. Let us now see that this is not possible, obtaining the contradiction we are looking for.

Indeed, since $a_{1}$ has degree 2 in $A_{1}$ and it can appear in only one subgraph, it must have degree two in the subgraph in which it appears, i.e. in the middle of a $P_{3}$ or a $P_{4}$, because $A_{1}$ is triangle-free. It is easy to see that this is equivalent to partitioning the edges of the graph $H$ depicted in FIG. 2 2 into subgraphs with at most 3 edges, in such a way that the thick edge $e$ appears in a subgraph with at most 2 edges, and each vertex appears in at most 2 subgraphs. Observe that $H$ is cubic and triangle-free. Let $n_{1}$ be the total number of vertices of degree 1 in all the subgraphs of the decomposition of $H$. Since each vertex of $H$ can appear in at most 2 subgraphs and $H$ is cubic, each vertex can appear with degree 1 in at most 1 subgraph. Thus, $n_{1} \leq|V(H)|=6$.

Since we have to use at least 1 subgraph with at most 2 edges and $|E(H)|=9$, there are at least $1+\left\lceil\frac{9-2}{3}\right\rceil=4$ subgraphs in the decomposition of $H$. But each subgraph involved in the decomposition of $H$ has at least 2 vertices of degree 1 , because $H$ is triangle-free. Therefore, $n_{1} \geq 8$, a contradiction.

\subsection{Case $C \geq 5$}

For $C \geq 5$ we can easily prove that $M(C, 3)=2$, making use of a conjecture made by Bermond et al. in 1984 [5] and proved by Thomassen in 1999 [19]: 
Theorem 2 ( [19]). The edges of a cubic graph can be 2-colored such that each monochromatic component is a path of length at most 5.

A linear $k$-forest is a forest consisting of paths of length at most $k$. The linear $k$-arboricity of a graph $G$ is the minimum number of linear $k$-forests required to partition $E(G)$, and is denoted by $l a_{k}(G)[5$. Theorem 2 is equivalent to saying that, if $G$ is cubic, then $l a_{5}(G)=2$.

Let us now see that Theorem 2 implies that $M(C, 3)=2$ for all $C \geq 5$. Indeed, all the paths of the linear forests have at most 5 edges, and each vertex will appear in exactly 2 linear 5 -forests, so the decomposition given by Theorem [19] is a partition of the edges of a cubic graph into subgraphs with at most 5 edges, in such a way that each vertex appears in at most 2 subgraphs. In fact the result of [19] is stronger, in the sense that $G$ can be any graph of maximum degree at most 3 . Thus, we deduce that

Corollary 2. For any $C \geq 5, M(C, 3)=2$.

Thomassen also proved [19] that 5 cannot be replaced by 4 in Theorem 2, This fact do not imply that $M(4,3)=3$, because of the following reasons: (i) the subgraphs of the decomposition of the request graph are not restricted to be paths, and (ii) it is not necessary to be able to find a 2-coloring of the subgraphs of the decomposition (a coloring in this context means that each subgraph receives a color, and 2 subgraphs with the same color must have empty intersection).

\section{Conclusions}

We considered the traffic grooming problem in unidirectional WDM rings when the request graph belongs to the class of graph with maximum degree $\Delta$. This formulation allows the network to support dynamic traffic without reconfiguring the electronic equipment at the nodes. We formally defined the problem, and we focused mainly on the cases $\Delta=2$ and $\Delta=3$, solving completely the former and solving all the cases of the latter, except the case when the grooming value $C$ equals 4. We proved in Section 4.1 that $M(3,3)=3$, and in Section 4.2 that $M(C, 3)=2$ for all $C \geq 5$. Proposition 3 states that $M(4,3, \mathcal{C})=2, \mathcal{C}$ being the

Table 1. Values of $M(C, \Delta)$. The case $C=4, \Delta=3$ is a conjectured value.

\begin{tabular}{|c|c|c|c|c|c|c|c|c|c|}
\hline$C|\Delta|$ & 1 & 2 & 3 & 4 & 5 & 6 & & & $\Delta$ \\
\hline \begin{tabular}{|l||}
1 \\
\end{tabular} & 1 & 2 & 3 & 4 & 5 & 6 & $\ldots$ & & $\Delta$ \\
\hline 2 & 1 & 2 & 3 & $\geq 3$ & $\geq 4$ & $\geq 5$ & & $\geq$ & $\frac{3 \Delta}{4}$ \\
\hline 3 & 1 & 2 & 3 & $\geq 3$ & $\geq 4$ & $\geq 4$ & $\ldots$ & $\geq$ & $\frac{2 \Delta}{3}$ \\
\hline 4 & 1 & 2 & $2 ?$ & $\geq 3$ & $\geq 4$ & $\geq 4$ & . & $\geq$ & $\frac{5 \Delta}{8}$ \\
\hline 5 & 1 & 2 & 2 & $\geq 3$ & $\geq 3$ & $\geq 4$ & $\ldots$ & $\geq$ & $\frac{3 \Delta}{5}$ \\
\hline$\ldots$ & . & . & . & $\ldots$ & $\ldots$ & $\ldots$ & $\ldots$ & & $\ldots$ \\
\hline$C$ & 1 & 2 & 2 & $\geq 3$ & $\frac{C+1}{C} \frac{5}{2}$ & $\geq 4$ & . & $\geq$ & $\frac{C+1}{C} \frac{\Delta}{2}$ \\
\hline
\end{tabular}


class of cubic graph with a perfect matching. Because of the integrality of $M(C, \Delta)$ and Lemma 3, $M(4,3)$ equals either 2 or 3 . We conjecture that $M(4,3)=2$.

We also deduced lower and upper bounds in the general case (any value of $C$ and $\Delta$ ). Table 1 summarizes the values of $M(C, \Delta)$ that we obtained.

This problem can find wide applications in the design of optical networks using WDM technology. It would be interesting to continue the study for larger values of $\Delta$, which will certainly rely on graph decomposition results. Another generalization could be to restrict the request graph to belong to other classes of graphs for which there exist powerful decomposition tools, like graphs with bounded tree-width, or families of graphs excluding a fixed graph as a minor.

Acknowledgement. The authors want to thank the anonymous referees for very helpful remarks that have substantially improved the paper.

\section{References}

1. Amini, O., Pérennes, S., Sau, I.: Hardness and Approximation of Traffic Grooming. In: Tokuyama, T. (ed.) ISAAC 2007. LNCS, vol. 4835, pp. 561-573. Springer, Heidelberg (2007)

2. Bermond, J.-C., Coudert, D.: Traffic grooming in unidirectional WDM ring networks using design theory. In: IEEE ICC, Anchorage, Alaska, vol. 2, pp. 1402-1406 (May 2003)

3. Bermond, J.-C., Coudert, D.: The CRC Handbook of Combinatorial Designs, 2nd edition. In: Colbourn, C.J., Dinitz, J.H. (eds.) Discrete Mathematics and Its Applications, ch. VI.27, Grooming, 2nd edn. Discrete Mathematics and Its Applications, vol. 42, pp. 493-496. CRC Press, Boca Raton (2006)

4. Bermond, J.-C., Coudert, D., Muñoz, X.: Traffic grooming in unidirectional WDM ring networks: the all-to-all unitary case. In: The 7th IFIP Working Conference on Optical Network Design \& Modelling - ONDM 2003, pp. 1135-1153 (February 2003)

5. Bermond, J.-C., Fouquet, J.-L., Habib, M., Péroche, B.: On linear $k$-arboricity. Discrete Math. 52(2-3), 123-132 (1984)

6. Berry, R., Modiano, E.: Reducing electronic multiplexing costs in SONET/WDM rings with dynamically changing traffic. IEEE J. on Selected Areas in Comm. 18, 1961-1971 (2000)

7. Chiu, A.L., Modiano, E.H.: Traffic grooming algorithms for reducing electronic multiplexing costs in WDM ring networks. IEEE/OSA Journal of Lightwave Technology 18(1), 2-12 (2000)

8. Dutta, R., Rouskas, N.: On optimal traffic grooming in WDM rings. IEEE Journal of Selected Areas in Communications 20(1), 1-12 (2002)

9. Dutta, R., Rouskas, N.: Traffic grooming in WDM networks: Past and future. IEEE Network 16(6), 46-56 (2002)

10. Erdös, P., Sachs, H.: Reguläre graphe gegebener taillenweite mit minimaler knotenzahl. Wiss. Z. Martin-Luther-Univ. Halle-Wittenberg Math.-Natur. Reihe 12, 251-257 (1963)

11. Flammini, M., Monaco, G., Moscardelli, L., Shalom, M., Zaks, S.: Approximating the traffic grooming problem in tree and star networks. In: Fomin, F.V. (ed.) WG 2006. LNCS, vol. 4271, pp. 147-158. Springer, Heidelberg (2006) 
12. Flammini, M., Moscardelli, L., Shalom, M., Zaks, S.: Approximating the traffic grooming problem. In: Deng, X., Du, D.-Z. (eds.) ISAAC 2005. LNCS, vol. 3827, pp. 915-924. Springer, Heidelberg (2005)

13. Gerstel, O., Lin, P., Sasaki, G.: Wavelength assignment in a WDM ring to minimize cost of embedded SONET rings. In: IEEE Infocom, San Francisco, California, pp. 94-101 (1998)

14. Gerstel, O., Ramaswani, R., Sasaki, G.: Cost-effective traffic grooming in WDM rings. IEEE/ACM Transactions on Networking 8(5), 618-630 (2000)

15. Goldschmidt, O., Hochbaum, D., Levin, A., Olinick, E.: The SONET edge-partition problem. Networks 41(1), 13-23 (2003)

16. Modiano, E., Lin, P.: Traffic grooming in WDM networks. IEEE Communications Magazine 39(7), 124-129 (2001)

17. Petersen, J.P.: Die Theorie der Regulären Graphs (The Theory of Regular Graphs). Acta Mathematica 15, 193-220 (1891)

18. Somani, A.: Survivable traffic grooming in WDM networks. In: Gautam, D.K. (ed.) Broad band optical fiber communications technology - BBOFCT, Jalgaon, India, December 2001, pp. 17-45. Nirtali Prakashan (2001) (Invited paper)

19. Thomassen, C.: Two-coloring the edges of a cubic graph such that each monochromatic component is a path of length at most 5. J. Comb. Theory Ser. B 75(1), 100-109 (1999)

20. Wan, P.-J., Calinescu, G., Liu, L., Frieder, O.: Grooming of arbitrary traffic in SONET/WDM BLSRs. IEEE Journal of Selected Areas in Communications 18(10), 1995-2003 (2000)

21. Wang, J., Cho, W., Vemuri, V., Mukherjee, B.: Improved approches for costeffective traffic grooming in WDM ring networks: Ilp formulations and single-hop and multihop connections. IEEE/OSA Journal of Lightwave Technology 19(11), $1645-1653$ (2001)

22. Zhu, K., Mukherjee, B.: A review of traffic grooming in WDM optical networks: Architectures and challenges. Optical Networks Magazine 4(2), 55-64 (2003) 